\title{
Cosmic ray measurements in the atmosphere at several latitudes in October, 2014
}

\author{
Vladimir Makhmutov*, Galina Bazilevskaya, Yuri Stozhkov, Maxim Philippov \\ Lebedev Physical Institute RAS, 119991, Moscow, Leninsky pr.53, Russia \\ E-mails: makhmutvesci.lebedev.ru,bazilevsesci.lebedev.ru, \\ stozhkovdfian.fiandns.mipt.ru, mfilippovdfrtk.ru
}

\section{Yoav Yair, Roy Yaniv}

Interdisciplinary Center, IDC, Herzliya, Israel

E-mail: yoav.yairdidc.ac.il

Department of Geosciences, Tel Aviv University, Tel Aviv, Israel

E-mail: royyaniv78@gmail.com

\section{Giles Harrison, Keri Nicoll}

Department of Meteorology, University of Reading P.O. Box 243, Earley Gate, Reading, Berks, RG6 6BB UK

E-mails: r.q.harrisondreading.ac.uk, k.a.nicollereading.ac.uk

\section{Karen Aplin}

Department of Physics, Denys Wilkinson Building, Keble Road, Oxford OX1 3RH UK

E-mail: karen.aplin@physics.ox.ac.uk

Cosmic ray fluxes in the atmosphere were recorded during balloon flights in October 2014 in northern Murmansk region, Apatity (Russia; 67 $33^{\prime} \mathrm{N}, 33^{\circ} 24^{\prime} \mathrm{E}$ ), in Antarctica (observatory

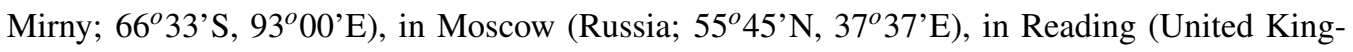

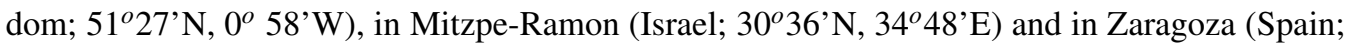
$\left.41^{\circ} 9^{\prime} \mathrm{N}, 0^{\circ} 54^{\prime} \mathrm{W}\right)$. Two type of cosmic ray detectors were used, namely, (1) the standard radiosonde and its modification constructed at the Lebedev Physical Institute (Moscow, Russia) and (2) the device manufactured at the Reading University (Reading, United Kingdom). We compare and analyze obtained data and focus on the estimation of the cosmic ray latitudinal effect in the atmosphere.

The 34th International Cosmic Ray Conference,

30 July- 6 August, 2015

The Hague, The Netherlands

\footnotetext{
*Speaker.
} 


\section{Introduction}

Since the International Geophysical Year (1957) the Lebedev Physical Institute (LPI) has performed charge particle flux measurement in the atmosphere [U]. The observations take place at the polar and mid-latitudes onboard the meteorological balloons. More than 83000 balloon flights were carried out up to now. The project is mainly aimed at the study of galactic and solar cosmic ray temporal and spatial variations. The magnetospheric electron precipitations are also recorded at the northern polar latitude. During the last decades the results of the LPI cosmic ray balloon observation are a subject of growing interest of specialists in atmospheric processes, e.g., [2], 目, 目, 回, 目]. Actually cosmic rays are the main source of ionization at the heights of $\approx 4-30 \mathrm{~km}$, the role of solar cosmic rays spreads much higher, therefore they influence significantly on neutral gas-phase chemistry, ozone layer and atmospheric electricity [ $[\mathbf{D}]$. It is very important to develop and upgrade charge particle monitoring in the atmosphere. The University of Reading has undertaken first steps in this direction [8]. In 2011 the International Space Science Institute (ISSI) supported the project "Study of cosmic ray influence upon atmospheric processes" (Leader I. Mironova). Then a collaboration between the LPI and the University of Reading was initiated. On 22-24 October 2014 we have performed a series of balloon flights including LPI and Reading devices onboard the same balloon. The launches were made at the Arctic and Antarctic, Moscow (Russia), Reading (UK), Zaragoza (Spain) and Mitzpe-Ramon (Israel). Here we present the preliminary results of the joint flights and the dependence of charged particle flux on latitude which is compared with previously made observations.

\section{Instrumentation}

Two kind of cosmic ray devices constructed at the Reading University (United Kingdom; RDG-device) and at the Lebedev Physical Institute (Russia; LPI-device) were used in the joint balloon experiment. Also, regular balloon cosmic ray measurements by standard radiosondes were done at Mirny, Murmansk and Moscow in October, 2014.

\subsection{LPI cosmic ray device}

Standard radiosondes launched on the rubber meteorological balloons with diameter of $1.5 \mathrm{~m}$ were used in the regular cosmic ray measurements in the atmosphere at Apatity (Arctica), Mirny (Antarctica) and Moscow according to their duty-cycle schedule on 22 and 24 October 2014. The standard radiosonde includes a detector of charged particles, a sensor of atmospheric pressure, electronic scheme with a radio transmitter, high voltage supply, and power supply batteries. The detector consists of two Geiger counters of STS-6 type to detect omnidirectional and vertical fluxes of particles ([四], and references therein). A STS-6 Geiger tube is $98 \mathrm{~mm}$ effective length and 19 $\mathrm{mm}$ in diameter. The thickness of steel walls equals $50 \mathrm{mg} \cdot \mathrm{cm}^{-2}$. Energy cutoff of detected particles is $\mathrm{E}_{e}=200 \div 300 \mathrm{keV}$ for electrons and $\mathrm{E}_{p}=5 \mathrm{MeV}$ for protons. The telescope has a $7-\mathrm{mm}$ $\mathrm{Al}$ absorber between counters, which gives with account of the counter walls the energy cutoff $\mathrm{E}_{e}=5 \mathrm{MeV}$ for electrons and $\mathrm{E}_{p}=30 \mathrm{MeV}$ for protons. The geometrical factors of a single counter $\mathrm{G}_{c}$ and of a telescope $\mathrm{G}_{t}$ depend on the angular distribution of detected particles. For isotropic 
angular distribution of particles in the upper hemisphere these values are equal to $\mathrm{G}_{c}=15.1 \mathrm{~cm}^{2}$ and $\mathrm{G}_{t}=17.8 \mathrm{~cm}^{2} \cdot \mathrm{sr}$.

For the cosmic ray measurements in Spain a modification of the standard radiosonde was used. It was appended with the pressure and temperature sensors, SD memory card for data record. Also it has a microcontroller for counting and sampling pulses by the time, reading and processing pressure and temperature, writing the data to the SD card (Figure 1). The LPI and RDG cosmic ray devices were recovered after flight on October 24 and recorded data were obtained from the SD cards.

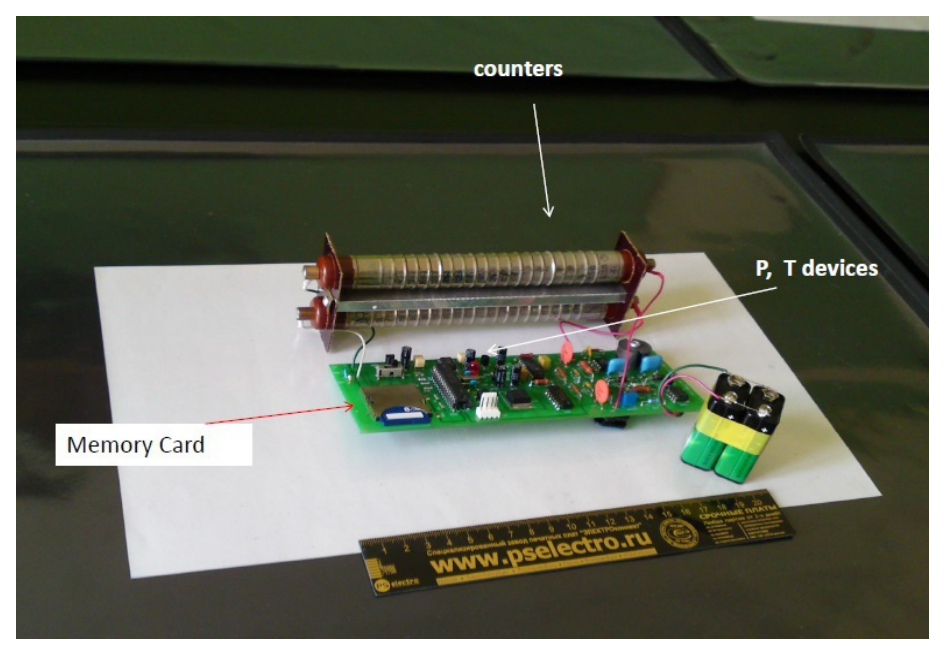

Figure 1: LPI cosmic ray device based on two Geiger counters STS-6, pressure and temperature sensors $(\mathrm{P}, \mathrm{T})$, crystal oscillator for timing and counting, and SD memory card for data record.

\subsection{RDG cosmic ray device}

The Reading device (RDG) consists of a battery-derived high voltage supply, which provides bias to two LND714 Geiger tubes arranged as a telescope [ [ 8$]$. The device is displayed in Figure ㅁ. The LND714 Geiger tube is $15.9 \mathrm{~mm}$ length and $4.83 \mathrm{~mm}$ in diameter. It is mainly sensitive

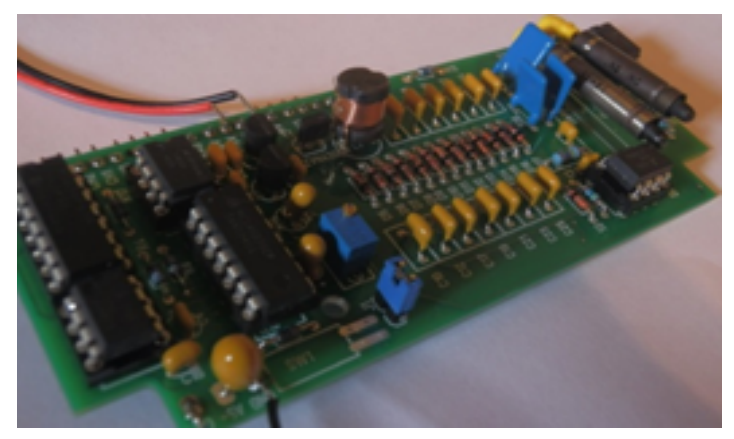

Figure 2: RDG cosmic ray device with two miniature Geiger tubes of LND714 type. The device can be incorporated into the standard meteorological radiosonde.

to gamma radiation. Pulses from the tubes are counted and time-stamped by a programmed microcontroller, and sent to the ground station by the UHF radio link, combined with the standard 
meteorological measurements and the GPS receiver. There are also an internal real time clock, temperature sensor and micro SD card. Great advantage of RDG is that it is developed as a package for easy attachment to meteorological radiosondes. That is RDG can be launched at many different sites of the globe. A disadvantage is that the sampling volume is small, which means that the count rate is also small. In general this drawback may be partly compensated by the frequent launching.

Note, that only the data of omnidirectional cosmic ray flux measurements with both LPI and RDG devices were used in this work.

\section{Data of observations}

Several balloon flights with the LPI and RDG sensors were performed on 22 and 24 October 2014. Table describes the launching sites with their geographic coordinates and geomagnetic cutoff rigidity values $\mathrm{Rc}(\mathrm{GV})$. Also a type of device is given. The flights were made according to the duty-cycle schedule of the regular LPI ballooning, so the data are added by the results of LPI measurements at the permanent sites of observation: Mirny, Apatity and Moscow. The results of LPI measurements on 24 October 2014 are given in Figure B]. The latitude dependence of the Geiger tube count rate is clearly seen.

Table 1: Balloon cosmic ray measurements in the atmosphere in October, 2014

\begin{tabular}{|c|c|c|c|c|}
\hline Location & Coordinates & $\mathrm{Rc}(\mathrm{GV})$ & 22.10 .2014 & 24.10 .2014 \\
\hline Mirny(Antarctica, RU) & $66^{\circ} 33^{\prime} \mathrm{S}, 93^{\circ} 00^{\prime} \mathrm{E}$ & 0.03 & LPI & LPI \\
Apatity (RU) & $67^{\circ} 33^{\prime} \mathrm{N}, 33^{\circ} 24^{\prime} \mathrm{E}$ & 0.56 & LPI & LPI \\
Moscow (RU) & $55^{\circ} 45^{\prime} \mathrm{N}, 37^{\circ} 37^{\prime} \mathrm{E}$ & 2.36 & LPI & LPI \\
Reading (UK) & $51^{\circ} 27^{\prime} \mathrm{N}, 0^{\circ} 58^{\prime} \mathrm{W}$ & 3.6 & RDG & \\
Zaragoza (Spain) & $41^{\circ} 9^{\prime} \mathrm{N}, 0^{\circ} 54^{\prime} \mathrm{W}$ & 4.6 & & RDG, LPI \\
Mitzpe-Ramon (Israel) & $30^{\circ} 36^{\prime} \mathrm{N}, 34^{\circ} 48^{\prime} \mathrm{E}$ & 10.3 & RDG & \\
\hline
\end{tabular}

During the flight at Zaragoza on 24 October 2014 the LPI and RDG devices were lifted by the same balloon. This enable us to adjust the RDG records to the LPI ones. Correspondence between the count rates of the both devices is presented in the left panel of Figure $⿴$. The scatter may be due to different efficiency of devices to particle types. For instance, the LPI device records the gamma radiation only with efficiency of $<1 \%$ while the RDG tubes are designed to measure gamma ray flux. In the bulk of atmosphere the charged particle and gamma fluxes are in equilibrium to the first approximation e.g. [Q], but future work will simulate the response of the two different measurement approaches more fully. At the moment we took a dependence shown in the left panel of Figure 9 to adjust the records of the RDG device to the LPI ones. Right panel of Figure $⿴$ depicts the result of adjustment. Afterwards the same adjustment was applied to all other flights of the RDG device shown in Table 1.

Figure 5 presents the results of charged particle observations on 22 and 24 October 2014. The count rates of the RDG devices are adjusted to the LPI device. The observations cover the geomagnetic cut-off rigidity range between $0.03 \mathrm{GV}$ and $10.3 \mathrm{GV}$, so it is interesting to compare the results with data obtained in the stratosphere during a previous latitude survey performed at the time period close to the solar activity maximum. 


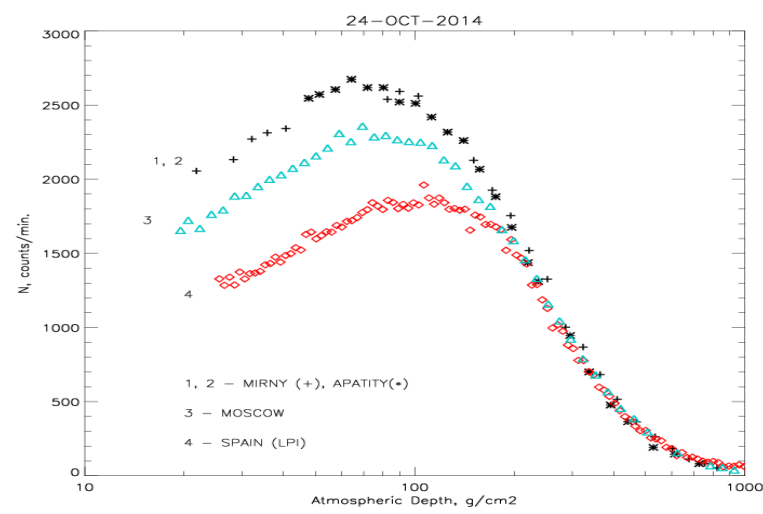

Figure 3: Cosmic ray measurements by standard radiosondes ( $\mathrm{N}$-count rate per minute) at Mirny, Moscow and Apatity on October 24, 2014 indicated by 1, 2 and 3. The LPI cosmic ray device recorded during balloon flight in Zaragoza are shown by 4.
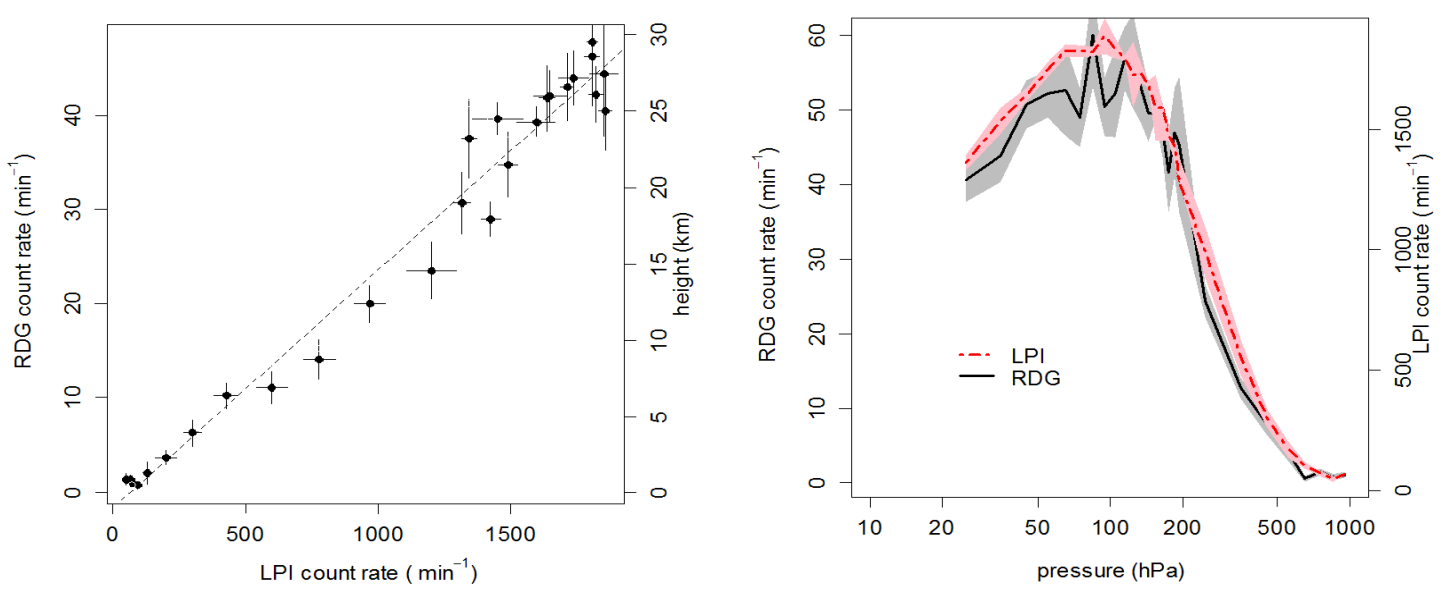

Figure 4: Left: Count rates from Reading instrument (y-axis) plotted against Lebedev instrument (x-axis). Points represent the mean count rate in $1 \mathrm{~km}$ intervals from individual 1 min count rates, with uncertainty bars representing 1.96 standard errors in the mean. The dashed line represents an (unweighted) least-squares regression line fitted to this data $[\mathrm{y}=(0.0252 \pm 0.008) \mathrm{x}+(-1.6082 \pm 1.0454)]$. Height has been added as a secondary y-axis. Right: Count rates obtained from the Reading (solid line) and Lebedev (dashed line) devices carried on the same balloon, plotted against pressure measurements made by a Bosch BMP180 sensor. For both counters, the $1 \mathrm{~min}$ count rate values available were averaged between pressure levels of 20, $30,40,50,60,70,80,90,100,110,120,130,140,150,160,170,180,190,200,300,400,500,600,700$, $800,900,1000$, and $1100 \mathrm{hPa}$. (The shaded bands in each case represent a range of 1.96 standard errors in the mean value obtained.)

The charged particle measurements in the stratosphere at locations with different geomagnetic cutoff rigidities were fulfilled by LPI during the sea expeditions in 1968-1970 [ए]]. The solar activity was rather greater $(\mathrm{Rz} \simeq 100)$ than in October $2014(\mathrm{Rz} \simeq 60)$. The solar magnetic field was just after reversal from negative to positive (in the northern solar hemisphere) in the both periods. Since the accuracy of measurements in 2014 is not high we can only look at the latitude dependence of the charged particle fluxes in the Regener-Pfotzer maximum where the statistics are better. Keeping in mind difference in solar activity the agreement between the results of 1968- 


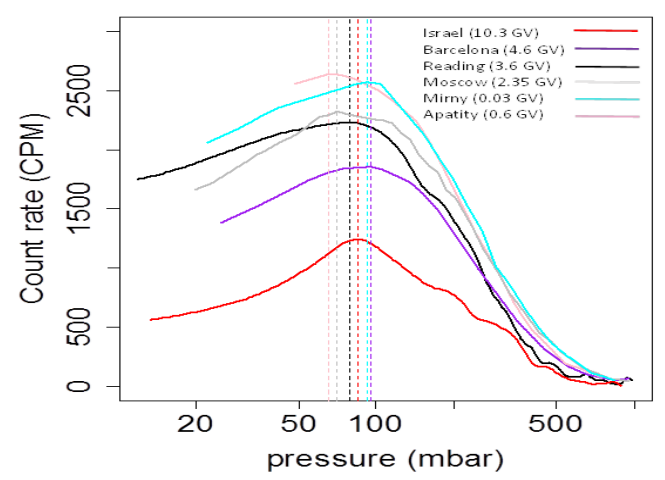

Figure 5: The count rates vs. atmospheric depth for balloon flights at various latitudes on 22 and 24 October 2014. The results of the RDG devices are adjusted to the LPI device as in Figure 4. Dotted lines indicates the Regener-Pfotzer maximum region.

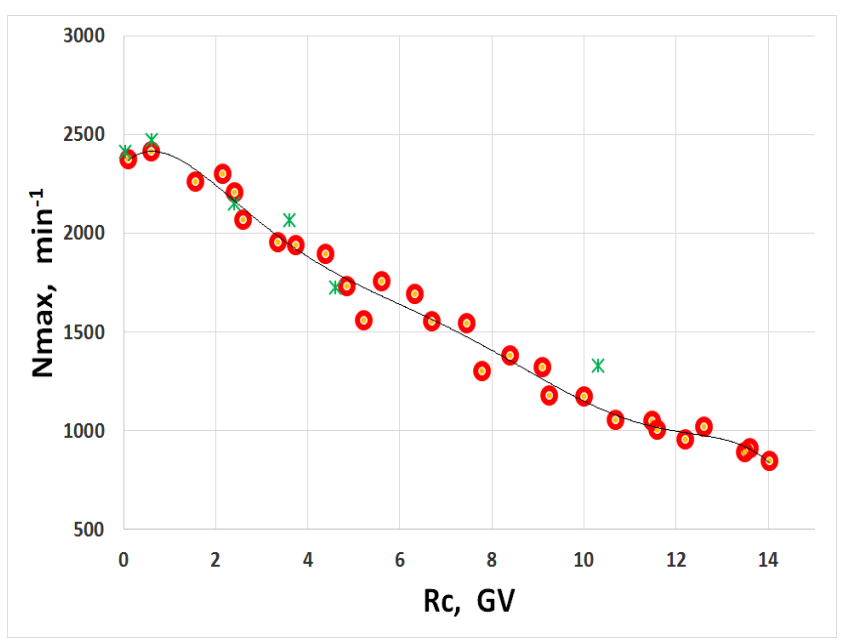

Figure 6: Count rates of the LPI cosmic ray device in the Regener-Pfotzer maximum in the stratosphere versus the geomagnetic cutoff values. Circles are the results of the sea expedition in 1968-1970 [ए]]. Solid curve is a polinomial fit of these data. Asterisks are the results of observations in October 2014 where the data of the RDG device are adjusted to the LPI as shown in Figure 4.

1970 and 2014 is quite satisfactory. It is seen in Figure $\mathbf{6}$ where the data of 2014 are indicated by asterisks.

\section{Discussion}

Monitoring of the charged particle fluxes enables direct calculation of ionisation in the atmosphere. The ion production rates in the atmosphere can be easily obtained from the recorded particle flux. Empirical relation between the two parameters was found in [ए0] based on comparison between the results of the measurements of ion production rates in the atmosphere by Neher [122, [3]] and the LPI results of charged particle fluxes measurements in 1958-1965. The relation is valid at altitudes of $5-35 \mathrm{~km}$. It is only slightly dependent on cutoff rigidity and solar activity level. Figure $\square$ demonstrates the charged particle fluxes and ion production rate obtained from the 
balloon flights in October 2014. For comparison the cosmic ray induced ionization was estimated using procedure [144, [5]]. It is seen that the results of observations and calculations are not quite consistent. Probably the data of 1 day observations fluctuate, so do not accurate represent the monthly averaged ionization which is given by the model [14, [5]. On the other hand, the model [패, ㄷ]] gives significant latitude effect around $12-13 \mathrm{~km}$ which is actually not observed.

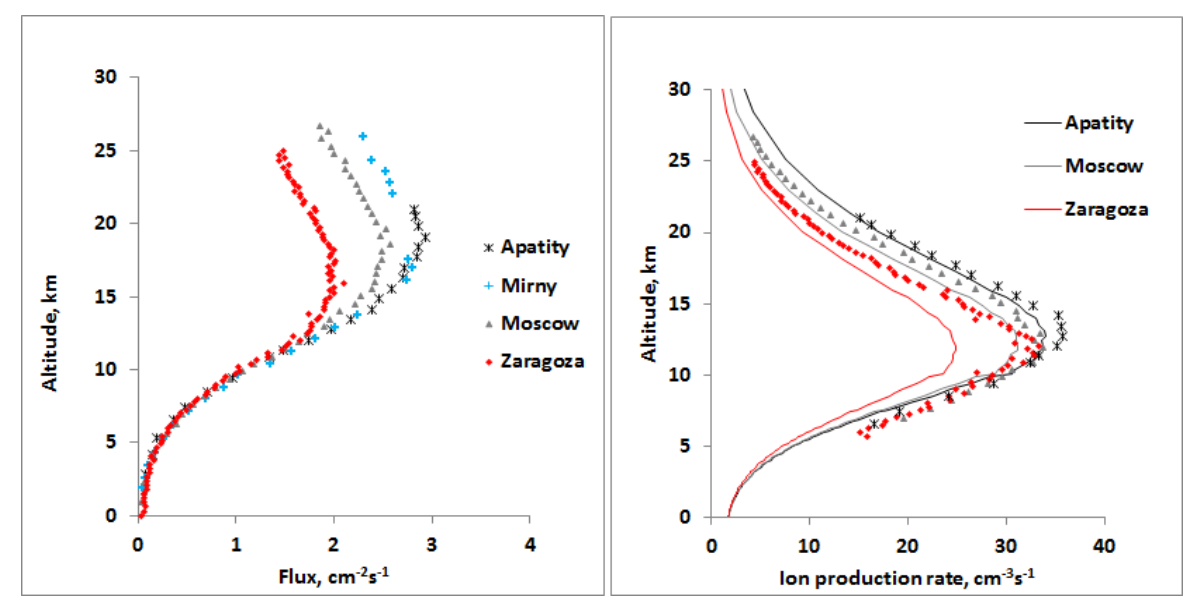

Figure 7: Altitude profiles of cosmic ray fluxes determined at different geomagnetic locations (left panel) and ion production rates (right panel) determined from results of cosmic ray measurements in October, 2014. The solid curves are the results of estimation of the monthly mean ion production rate by the model [144, [5]].

\section{Summary}

The observations of charged particle fluxes in the atmosphere are under development at Reading University. Hopefully, the observations will continue and complement the long-term LPI experiment. The first joint balloon flight with the devices of RDG and LPI onboard has shown that the results of both devices can be reconciled however there are many points to work on. Monitoring of energetic radiation in the atmosphere is the only way to verify the models describing the response of atmosphere to ionization and understand the underlying physics.

\section{Acknowledgments}

This work would not be possible without support of the International Space Science Institute (ISSI). It was initiated due to the ISSI grant in 2011 "Study of cosmic ray influence upon atmospheric processes" and continued in frame of the ISSI grant in 2014 "Specification of ionization sources affecting atmospheric processes" (both grants under leadership of Irina Mironova). The LPI people acknowledge support of the RFBR grants 14-02-00905a, 14-02-10006k, 13-0200585, 13-02-00931, 15-02-10070k. The radiosonde work at Reading was supported by NERC (NE/H002081/1) and STFC (ST/K001965/1). Support for Y. Yair and R. Yaniv was received from The Israeli Science Foundation grant 423/13. 


\section{References}

[1] Yu.I. Stozhkov, N.S. Svirzhevsky, G.A. Bazilevskaya, A.N. Kvashnin, V.S. Makhmutov, A.K. Svirzhevskaya, Long-term (50 years) measurements of cosmic ray fluxes in the atmosphere, Adv. in Space Res. 4410 (2009) 1124-1137

[2] A. Krivolutsky, A. Kuminov and T. Vyushkova, Ionization of the atmosphere caused by solar protons and its influence on ozonosphere of the Earth during 1994-2003, J. Atmos. Sol. Terr. Phys. 67 (2005) 105-117

[3] J. Kazil, E.R. Lovejoy, M.C. Barth, and K. O'Brien, Aerosol nucleation over oceans and the role of galactic cosmic rays, Atmos. Chem. Phys. 6 (2006) 4905-4924

[4] M. Calisto, I. Usoskin, E. Rozanov and T. Peter, Influence of Galactic Cosmic Rays on atmospheric composition and dynamics, Atmos. Chem. Phys. 11 (2011) 4547-4556

[5] M.J.Rycroft, K.A. Nicoll, K.L. Aplin, R.G. Harrison, Recent advances in global electric circuit coupling between the space environment and the troposphere, JASTP 90-91 (2012) 198-211

[6] I.A. Mironova and I.G. Usoskin, Possible effect of strong solar energetic particle events on polar stratospheric aerosol: a summary of observational results, Environ. Res. Lett. 9 (2014) 015002 (8pp)

[7] I.A. Mironova, K.L. Aplin, F. Arnold, G.A. Bazilevskaya, R.G. Harrison, A.A. Krivolutsky, K.A. Nicoll, E.V. Rozanov, E. Turunen, I.G. Usoskin,Energetic particle infuence on the Earth's atmosphere, Space Science Rev. accepted

[8] R.G. Harrison, K.A. Nicoll and A.G. Lomas, Note: Geiger tube coincidence counter for lower atmosphere radiosonde measurements, Rev. of Sci. Instr. 84 (2013) 076103

[9] L. Desorgher, E.O. Flueckiger, M. Gurtner, R. Buetikofer, Int. J. Modern Phys. 20(29) (2005) 6802-6804.

[10] G.A. Bazilevskaya, M.B. Krainev, V.S. Makhmutov, Effects of cosmic rays on the Earth's environment, Journal of Atmospheric and Solar-Terrestrial Physics 62(17-18) (2000) 1577-1586

[11] A.N. Charakhchyan, G.A. Bazilevskaya, Yu.I. Stozhkov, T.N. Charakhchyan, Cosmic rays in the stratosphere and in the near Earth space in the 19th and 20th cycles of solar activity in Trudy FIAN 88 3-50 Nauka Moscow (in Russian) 1976

[12] H.V. Neher, Cosmic ray particles that changed from 1954 to 1958 to 1965 Journal of Geophysical Research 72(5) (1967)1527-1539

[13] H.V. Neher, Cosmic rays at high latitudes and altitudes covering four solar maxima, Journal of Geophysical Research 76(7) (1971) 1637-1651

[14] I.G. Usoskin and G.A. Kovaltsov, Cosmic ray induced ionization in the atmosphere: Full modeling and practical applications, J. Geophys. Res. 111 (2006) D21206

[15] I.G.Usoskin, G.A.Kovaltsov,I.A. Mironova, Cosmic ray induced ionization model CRAC:CRII: An extension to the upper atmosphere, J. Geophys. Res. 115 (2010) D10302 